\title{
The Herbig B0e star HD 53367: circumstellar activity and evidence of binarity $\star \star \star \star$
}

\author{
M. A. Pogodin ${ }^{1,2}$, V. P. Malanushenko ${ }^{3,4}$, O. V. Kozlova ${ }^{3,4}$, T. N. Tarasova ${ }^{3,4}$, and G. A. P. Franco ${ }^{5}$ \\ 1 Pulkovo Observatory, Saint-Petersburg 196140, Russia \\ e-mail: pogodin@gao.spb.ru \\ 2 Isaac Newton Institute of Chile, Saint-Petersburg Branch, Russia \\ 3 Crimean Astrophysical Observatory, Nauchny, Crimea 334413, Ukraine \\ e-mail: [victor; oles; taya]@crao.crimea.ua \\ 4 Isaac Newton Institute of Chile, Crimean Branch, Ukraine \\ 5 Departamento de Física - ICEx - UFMG, Belo Horizonte, Brazil \\ e-mail: franco@fisica.ufmg.br
}

Received 27 June 2005 / Accepted 15 January 2006

\section{ABSTRACT}

\begin{abstract}
Aims. We investigate the spectroscopic behaviour of the young B0e star HD 53367 within a cooperative observing programme conducted from 1994 to 2005.

Methods. The data include more than 100 high-resolution spectra collected at the Crimean Astronomical Observatory (CrAO) near $\mathrm{H} \alpha, \mathrm{H} \beta, \mathrm{He}$ I $\lambda 5876,6678 \AA$, DNa I, and O II $\lambda 6641 \AA$ lines. Two spectra obtained at the Observatório do Pico dos Dias (LNA), in the spectral bands $\lambda \lambda$ 4575-4725 $\AA$ and $\lambda \lambda$ 5625-5775 $\AA$, were used for spectral classification of HD 53367. The temporal behaviour of the circumstellar lines $\mathrm{H} \alpha$ and $\mathrm{H} \beta$ as well as the photospheric lines O II $\lambda 6641 \AA$ and He I $\lambda 6678 \AA$ were investigated during different stages of the photometric activity of this object.

Results. We confirm that the long-term photometric variability of HD 53367 is related to the alternation of two states of this object when the gaseous circumstellar envelope disappears and rises again. Both these processes start near the star and spread to the outlying parts of the envelope. We find that the radial velocities of He I and O II photospheric lines demonstrate a cyclic variability with a period of $P=183.7$ days and semi-amplitude $K=19 \mathrm{~km} \mathrm{~s}^{-1}$. The radial velocity change is interpreted in the framework of a model in which the star is a component of an eccentric binary system. An orbital solution is derived and the system's parameters estimated. We find that the orbital eccentricity is $e=0.28$, and the mean companion separation is 1.7 AU.

Conclusions. Based on the estimated parameters, we conclude that the system consists of a massive $\left(\sim 20 M_{\odot}\right)$ main sequence primary B0e star, and a secondary which is most likely a 5 solar mass pre-main sequence object. We found evidence that the main part of the circumstellar gas in this system is concentrated near the secondary companion. Although the young age of HD 53367, its evolved primary B0e star seems to have already became a classical Be star exhibiting a specific alternation of the B-Be stages.
\end{abstract}

Key words. techniques: spectroscopic - stars: binaries: spectroscopic - stars: circumstellar matter - stars: emission-line, Be stars: individual: HD 53367 - stars: pre-main sequence

\section{Introduction}

HD 53367 (MWC 166, V750 Mon, B0e-B1e III-V, $V=7^{\mathrm{m}} 0$ ) is situated in the extended star formation region CMa R1 containing more then one hundred young stellar objects of different masses and an association of reflection nebulae. This object is likely to be the most massive $\left(15-25 M_{\odot}\right)$ and, consequently, the most evolved star among the objects of the region whose stellar composition was studied in detail by Tjin A Djie et al. (2001). HD 53367 is a visual binary system (RST 3489) with $\Delta m \sim 1.3$ and a separation between the components $\rho=0$.'6 ( $\sim 600 \mathrm{AU}-$ assuming the distance of about $1 \mathrm{kpc}$ suggested by Claria 1974).

This object is immersed in the dark nebula known as IC 2177 (Claria 1974) and displays emission lines in its visual spectrum, with $\mathrm{H} \alpha$ being the brightest, and its circumstellar environment shows signs of cool dust matter in the form of a far-IR

\footnotetext{
* Based on observations collected at the Crimean Astrophysical Observatory (CrAO, Ukraine), and Observatório do Pico dos Dias (LNA, Brazil).

$\star \star$ Table 1 is only available in electronic form at http://www. edpsciences.org
}

excess at $\lambda \geq 10 \mu \mathrm{m}$ (Tjin A Djie et al. 2001, and references therein). HD 53367 is among the original list of young Herbig Ae/Be stars (Herbig 1960) that are conventionally regarded as being pre-main sequence objects. Nevertheless, this star is very massive, and we know that such objects evolve directly from the earlier protostellar stages to the main sequence, completely escaping the intermediate pre-main sequence phase (see, for example, Palla \& Stahler 1993).

More then $200 U B V R$ photoelectric measurements of HD 53367 were collected over the last twenty years at the Mt. Maidanak Observatory (Tjin A Djie et al. 2001, and recent 2000-2004 data kindly put at our disposal by K. N. Grankin) showing that this is a photometrically active object. HD 53367 shows a long-term cyclic variability on the timescale of several years in the form of an alternation of two main states of brightness with $\Delta m \sim 0$. 25 . The colour-magnitude diagrams show that the colour indices $B-V$ and $V-R$ are bluer at minimum brightness while the dependence of the $U-B$ colour index is more complex. In order to explain the photometric behaviour of HD 53367, Tjin A Djie et al. (2001) suggested two mechanisms. The first one proposes that magnetic activity 


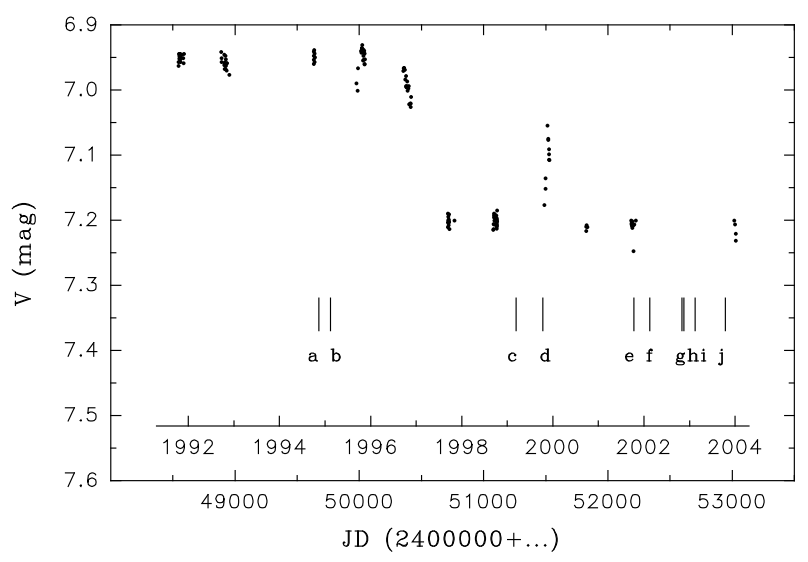

Fig. 1. The light variations of HD 53367 in the $V$-band measured at the Mt. Maidanak Observatory in 1991-2004. The data published by Tjin A Djie et al. (2001) are supplemented by recent 2001-2004 measurements by K. N. Grankin. This light curve covers the period of the most striking variability of the $\mathrm{H} \alpha$ and $\mathrm{H} \beta$ line profiles accompanying the transition of HD 53367 from the bright to the low photometric state. The vertical marks indicate the dates of the spectroscopic observations in relation to the profile variations displayed in Fig. 2: a) Nov. 16, 1994; b) Fev. 20, 1995; c) Mar. 23, 1999; d) Oct. 24, 1999; e) Oct. 26, 2001; f) Mar. 2, 2002; g) Nov. 18, 2002; h) Dec. 4, 2002; i) Mar. 1, 2003; j) Nov. 1, 2003. The spectra obtained on dates a) and b) were taken from Corporon \& Lagrange (1999).

in the stellar photosphere stimulates a cyclic development of cool spots on its surface. This leads to a decrease of the effective temperature causing the reddening of the star. This assumption, however, cannot explain why in this case the star becomes brighter. The second mechanism suggests the existence of a dense one-armed perturbation slowly precessing in the $\mathrm{CS}$ disk around a $\mathrm{Be}$ star. The periodic eclipse of this region together with the enhanced emission by the stellar limb results in the observed photometric and spectral variations. Figure 7 in the paper by Tjin A Djie et al. (2001) clearly illustrates that the emission component of the $\mathrm{H} \beta$ line is much stronger in the object's bright state than at its minimum of brightness. However, this phenomenon should be strictly periodic. The photometric curve of HD 53367 presented by Tjin A Djie et al. (2001) and its complement shown in our Fig. 1 do not allow us to conclude that this variability is periodic.

A third alternative that we believe to be a more realistic interpretation is that the observed variations of magnitudes and colours can be caused by the change of the amount of emitting gas in the circumstellar media.

Another remarkable property of HD 53367 is the significant radial velocity variations observed in a number of photospheric lines (Corporon \& Lagrange 1999, and references therein). This phenomenon has been investigated in detail by those authors. Based on 19 measurements of radial velocities of $\mathrm{He}$ I $\lambda 4471$, 5875, 6678 A lines obtained from May 1994 to January 1998, they determined a period of $P \approx 166$ days and an orbital eccentricity $e \approx 0.18$. However, besides the rather small number of measurements, the observational data cover only $30 \%$ of the period's phase, given that the maximum and the minimum positions of the orbital curve correspond to phases where observational points are absent. Therefore, their orbital solution requires further confirmation with a larger data sample.

The main purposes of the present work are: a) to collect new high-resolution spectroscopic data for HD 53367 in lines originating in the circumstellar envelope $(\mathrm{H} \alpha$ and $\mathrm{H} \beta)$ as well as in the stellar photosphere (O II $\lambda 6641 \AA$ and He I $\lambda 6678 \AA$ ); b) to compare the circumstellar spectrum of the object at different stages of its photometric activity and to test the alternative hypotheses proposed to explain its nature; c) to revise, on the basis of these new spectroscopic data, the earlier proposed orbital solution for HD 53367; and d) to estimate the parameters of the system's components.

\section{Observations}

The high-resolution spectroscopic observations of HD 53367 were carried out during several observing seasons from 1999 to 2005 at the Crimean Astrophysical Observatory (CrAO, Ukraine) and at the Observatório do Pico dos Dias (LNA/MCT, Brazil). The spectral resolving power was $(R \sim 20000)$ for CrAO and $(R \sim 15000)$ for LNA data. The $2.6 \mathrm{~m}$ Shajn telescope of $\mathrm{CrAO}$ was equipped with a Coudé spectrograph. The detector was a CCD camera SDS-9000 "Photometric GmbH" with 1024 pixel along the dispersion axis (until the middle of 2004). This equipment yields a wavelength coverage of about $65 \AA$ (near H $\alpha$ ). In October 2004 and March 2005 a P $8600770 \times 1152$ pixel CCD detector was used. The wavelength covered by this detector was about $30 \AA$.

During all observing seasons more than 100 spectra were obtained at $\mathrm{CrAO}$ near $\mathrm{H} \alpha, \mathrm{H} \beta, \mathrm{He} \mathrm{I} \lambda 5876,6678 \AA$, DNa I, and O II $\lambda 6641 \AA$ lines (see Table 1). The exposure times ranged from 15 to $60 \mathrm{~min}$ providing signal-to-noise ratio $S / N$ from 30 to 130 per resolving element (at the continuum level) depending on the CCD detector used, spectral region and weather conditions. Data reduction followed the standard procedures and was done with computer codes developed at the $\mathrm{CrAO}$ by S. G. Sergeev.

The observations at LNA were carried out using the Coudé spectrograph installed on the $1.6 \mathrm{~m}$ telescope and equipped with a 1024 pixel CCD detector along the dispersion axis. A wavelength covering about $150 \AA$ was achieved in each spectrum. The reduction process also followed the standard procedure and was done with the IRAF packages. Two spectra of HD 53367 were obtained at LNA on May 11, 2000 in the spectral bands $\lambda \lambda 4575-4725 \AA$ and $\lambda \lambda 5625-5775 \AA$ containing many absorption lines originating in the stellar photosphere (O I, O II, N II, C II, He I, He II, etc.), which can be used for spectral classification of the object.

Additionally, we used for comparison two échelle spectra of HD $53367(\lambda \lambda 3900-6800 \AA, R=42000)$ obtained in 1994-1998 by Corporon (Corporon \& Lagrange 1999) at the Observatoire de Haute-Provence (OHP, France) with the ÉLODIE spectrograph installed on the $1.93 \mathrm{~m}$ telescope (Table 2).

\section{Data analysis}

\subsection{The Balmer emission lines}

Our spectroscopic programme started in March 1999. It corresponds to two years after HD 53367 passed into its low photometric state, as one can see in Fig. 1.

The observed variations of the $\mathrm{H} \alpha$ and $\mathrm{H} \beta$ profiles collected on dates (a)-(j) are presented in Fig. 2. Theoretical photospheric profiles, calculated with the use of the SYNTH \& ROTATE code (Piskunov 1992), are given for comparison (dashed-line). The best fit of the synthetic spectrum was obtained by adjusting the spectrum collected on May 11, 2000 at LNA, in 


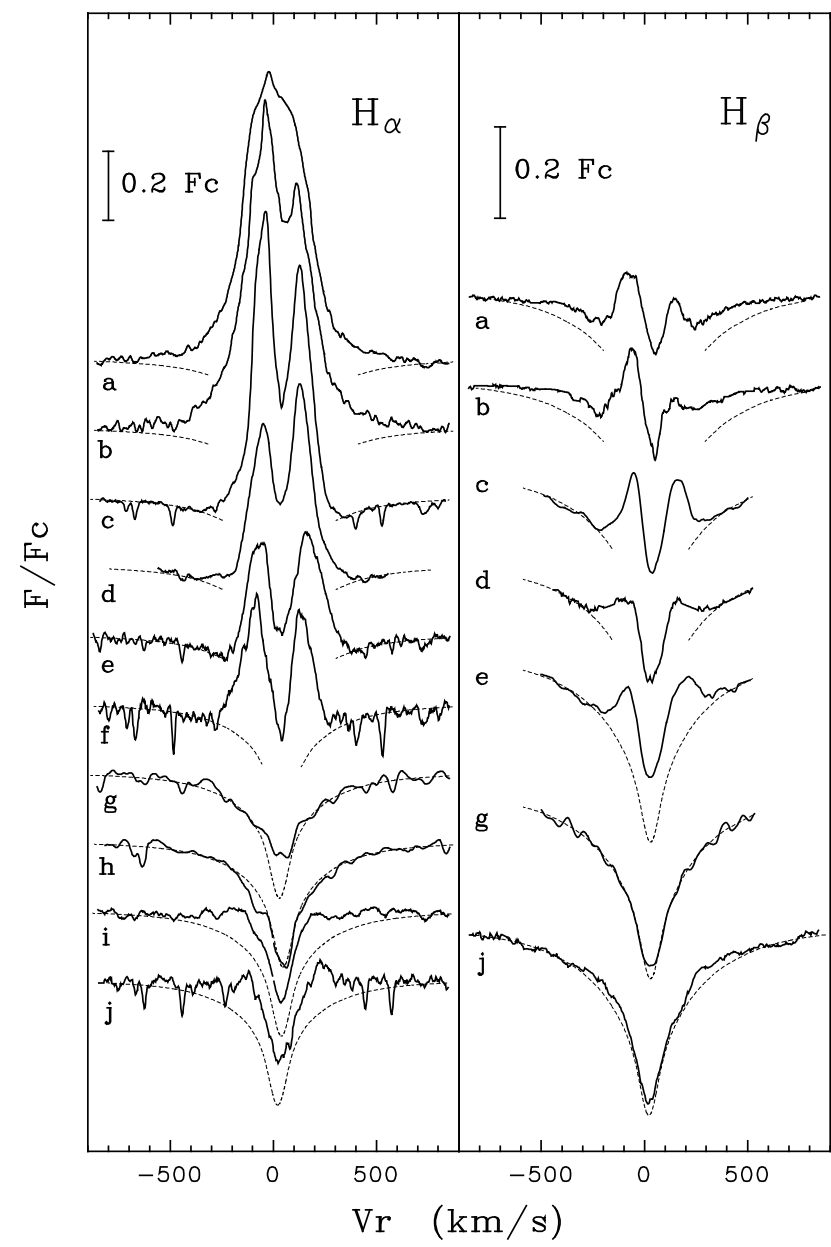

Fig. 2. Normalized profiles of the $\mathrm{H} \alpha$ and $\mathrm{H} \beta$ emission lines observed in the spectrum of HD 53367 on the dates marked in Fig. 1, when the object was passing from the bright to the low state of its brightness. The velocity scale is given with respect to the solar system. No attempt has been made to eliminate the water vapour lines overlapping the $\mathrm{H} \alpha$ profiles. Synthetic photospheric profiles are given for comparison (dashed lines). The parameters used to define the stellar model are given in the text. The synthetic profiles were shifted in accordance with the estimated stellar radial velocity for the corresponding dates (see Table 1).

Table 2. The $\log$ of the additional data obtained at the Observatoire de Haute-Provence (OHP, France) and at the Observatório do Pico dos Dias (LNA, Brazil).

\begin{tabular}{cccc}
\hline \hline Date & JD (2400000+) & Spectral region & Observatory \\
\hline Nov. 16, 1994 & 49673.60 & $\mathrm{H} \alpha \& \mathrm{H} \beta$ & OHP \\
Fev. 20, 1995 & 49769.45 & $\mathrm{H} \alpha \& \mathrm{H} \beta$ & OHP \\
May 11, 2000 & 51676.40 & $4575-4725 \AA$ & LNA \\
May 11, 2000 & 51676.43 & $5625-5775 \AA$ & LNA \\
\hline
\end{tabular}

the region $\lambda \lambda 4575-4725 \AA$, and corresponds to a model with the following parameters: $T_{\mathrm{eff}}=30000 \mathrm{~K}, \log g=4.2$, and $v \sin i=40 \mathrm{~km} \mathrm{~s}^{-1}$.

At the beginning of our observational series, HD 53367 was in its bright photometric state - profiles (a) and (b) shown in Fig. 2, which were observed on November 16, 1994 and February 20, 1995, respectively. These spectra were kindly put at our disposal by Corporon (Corporon \& Lagrange 1999). As one can see in Fig. 2, the $\mathrm{H} \alpha$ and $\mathrm{H} \beta$ emission profiles become progressively weaker from (a-b) to $(\mathrm{g}-\mathrm{h})$ dates when they disappear completely (by the end of 2002). In 2003 (i-j) emission starts to appear again in these lines. This is also seen in spectra obtained on later dates demonstrating slight irregular variations on a timescale of a month. Similar short-term variations of brightness are also observed on this occasion (see Fig. 1, December 1999 - January 2000 event).

Our analysis of the $\mathrm{H} \alpha$ and $\mathrm{H} \beta$ profiles observed for HD 53367 shows the following important properties:

1. The brightness decrease in HD 53367 is followed by a decay of emission in these lines. However, a significant difference exists in the timescales of these processes. The transition of HD 53367 from the bright to the low photometric states took little more than one year (from August 1996 to October 1997), while emission in Balmer lines was observed for five years more (until the end of 2002). It is even possible that the Balmer emission lines were already present in its decreasing phase at the end of 1994 (date "a"), namely two years before the object started dimming. We know, for example, that in 1981 the maximum relative intensity of the $\mathrm{H} \alpha$ profile achieved a value of 2.40 (Finkenzeller \& Mundt 1984), while at the end of 1994 it was only 1.90.

2. The decay of the Balmer emission lines in the object's spectrum was followed by a simultaneous decrease of intensity as well as of width of the line profile. Just before its disappearance the emission line profiles became rather weak and narrow (see date " $\mathrm{g}$ "). When a new Balmer emission started again (dates "i-j"), the $\mathrm{H} \alpha$ and $\mathrm{H} \beta$ lines were also weak in intensity, but the width of the lines was as large as in the bright photometric state (dates "a-b") and was equal to $\pm 700 \mathrm{~km} \mathrm{~s}^{-1}$.

\subsection{Radial velocities of the photospheric lines}

A regular positional variability of absorption lines originating in the stellar photosphere can be an important indicator of an object's binarity. Since we knew that HD 53367 showed evidence of being a binary system, we included a number of photospheric lines usable for radial velocity determination in our spectroscopic investigation of this star. For this purpose we chose lines whose contribution from the circumstellar matter to its photospheric profile were a minimum. After analysing the spectrum of HD 53367 we chose the following lines for radial velocity measurements: a) about 15 absorption lines in the spectral regions $\lambda \lambda 4575-4725 \AA$ and $\lambda \lambda 5625-5775 \AA$ obtained at LNA (see Fig. 3); b) the O II $\lambda 6641 \AA$ line which is one of the few detectable photospheric lines in the object's spectrum in its red region - where the CCD detectors installed at the Shajn telescope (CrAO) are more sensitive; and c) the He I $\lambda 6678 \AA$ line, which may be affected by circumstellar matter, but can be used to estimate the star's radial velocity, when the variable envelope does not contribute sufficiently to the line profile.

\subsubsection{The spectra collected at LNA}

Figure 3 presents the observed spectrum of HD 53367 obtained on May 11, 2000 at LNA in comparison with the corresponding best fitted synthetic spectrum. The main criteria used to achieve the fit were the width and the depth of the He II $\lambda 4686 \AA$ and He I $\lambda 4713 \AA$ line profiles. The observed spectrum on this date shows a regular velocity shift relative to the solar system of $+29.9 \pm 2.6 \mathrm{~km} \mathrm{~s}^{-1}$ (for 10 lines in the $\lambda \lambda 4575-4725 \AA$ region) and $+31.4 \pm 3.7 \mathrm{~km} \mathrm{~s}^{-1}$ (for 5 lines in the $\lambda \lambda 5625-5775 \AA$ region). 


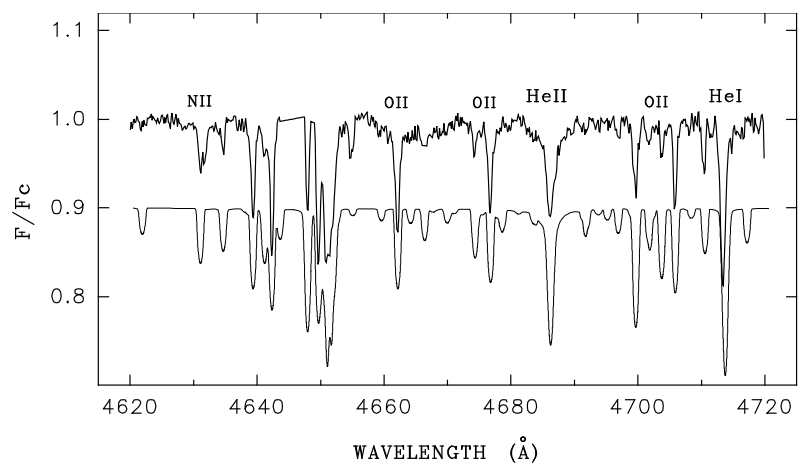

Fig. 3. The spectrum of HD 53367 in the region $\lambda \lambda 4575-4725 \AA$ obtained at the LNA on May 11, 2000. A synthetic theoretical spectrum is given for comparison. The corresponding model parameters used to obtain the synthetic spectrum are described in the text.

\subsubsection{The O $\| \lambda 6641 \AA$ line}

Positional variations of the O II $\lambda 6641 \AA$ line from date to date are illustrated in Fig. 4. The interstellar band centered at $\lambda 6614 \AA$ placed in the same spectrograms as the O II $\lambda 6641 \AA$ line is also shown to illustrate the positional stability of this feature with an accuracy of $\pm 0.5 \mathrm{~km} \mathrm{~s}^{-1}$ as a criterion of the high quality of the spectrograms containing the O II $\lambda 6641 \AA$ line and of their suitability for precise positional measurements. The radial velocity of this photospheric line was determined by measuring the center of gravity of the absorption profile.

Nevertheless the weakness of the O II $\lambda 6641 \AA$ line in the spectrum of HD 53367 was a limiting factor to the accuracy of the measurements. This was the main reason why we looked for another spectral line to measure the radial velocities of HD 53367. From an inspection of the HD 53367 spectrum, we chose He I $\lambda 6678 \AA$.

\subsubsection{The He $\mid \lambda 6678 \AA$ line}

The HeI $\lambda 6678 \AA$ line is much deeper than the O II $\lambda 6641 \AA$ line, but it is likely to be more affected by the circumstellar matter, even when the object is in its weakened envelope line emission state. Figure 5 displays some of the observed HeI $\lambda 6678 \AA$ profiles. They exhibit a complex and variable structure. Their depth and width are different on different dates. An additional wide shallow absorption is observed and a blueshifted emission peak appears episodically. Since the characteristic timescale of these variations is over months, they cannot be connected with rotation of inhomogeneities in the stellar surface. They are likely to be the result of the circumstellar influence on the photospheric profile. Nevertheless, a comparison with the synthetic model profile shows that the observed profiles always remain axially symmetric at the $0.85-0.95$ intensity level. We suppose that this component is mainly of CS origin, formed in the inner part of the disk, where the CS matter is axially symmetrically distributed relative to the star. Therefore, the velocity position of the component has to reflect the true orbital motion of the star itself. It can be measured as the bisector velocity of the line profile at the $0.9 F_{\mathrm{c}}$ level. The obtained values are presented in Table 1 as $V_{\mathrm{r}}$ of the He I $\lambda 6678$ Å line.

The analysis of the photospheric lines of HD 53367 provided a set of radial velocity measurements containing 76 data

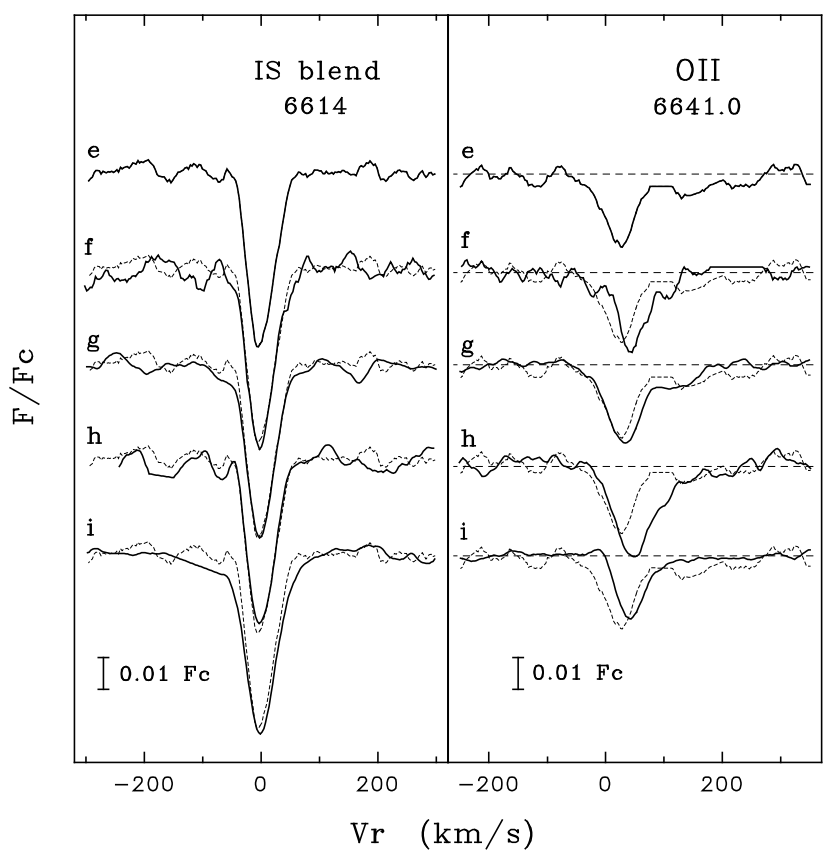

Fig. 4. The mean profiles of the O II $\lambda 6641 \AA$ line collected in observing seasons near the dates marked in Fig. 1 (right-hand panel): e) October 26-27, 2001 (4 measurements); f) March 1-5, 2002 (4 measurements); g) November 14-15, 2002 (2 measurements); h) December 4, 2002 (2 measurements); i) March 1-4, 2003 (5 measurements). The first profile e) overlaps each other profiles for comparison. The velocities measured on these dates are presented in Table 1. The mean velocity error is $\pm 2 \mathrm{~km} \mathrm{~s}^{-1}$. The horizontal dashed lines mark the continuum level $F_{\mathrm{c}}$. The velocity scale is given in the heliocentric rest frame. The left-hand panel illustrates the profiles of the interstellar band at $\lambda 6614 \AA$, observed in the same spectrograms as the O II $\lambda 6614 \AA$ profiles displayed in the right-hand panel, on the corresponding dates. The velocity scale is centered at the mean wavelength of the interstellar band for all observing seasons. The accuracy of the velocity measurements of this feature is better than $\pm 0.5 \mathrm{~km} \mathrm{~s}^{-1}$.

points ranging from $+19 \mathrm{~km} \mathrm{~s}^{-1}$ (October 2004) to $+69 \mathrm{~km} \mathrm{~s}^{-1}$ (December 2003). The positional variations of photospheric lines with so large an amplitude are consistent with the suggested binary nature of HD 53367.

\subsection{Orbital solution}

Given the assumption that HD 53367 is a spectroscopic binary system, we derived the properties of its components and its orbital elements from the obtained radial velocity curve.

With the aid of the new spectroscopic data we checked the period of 166 days suggested by Corporon \& Lagrange (1999). In the top panel of Fig. 6 we show what was obtained by combining our data (open circles), folded with the period of 166 days, with the data collected by Corporon \& Lagrange (1999) (filled triangles). As one can see, the new spectroscopic data exclude a $166^{\mathrm{d}}$-period. This failure led us to derive a new orbital solution based on the recent data. A least-square method was applied to calculate a new radial velocity curve and to determine the orbital elements (Tokovinin 1992). The obtained results are given in Table 3, and correspond to the orbital curve shown in the bottom panel of Fig. 6.

This solution corresponds to an eccentric orbit with the periastron oriented toward the observer. The orbital period has been derived with rather high accuracy, but the fact that its value 


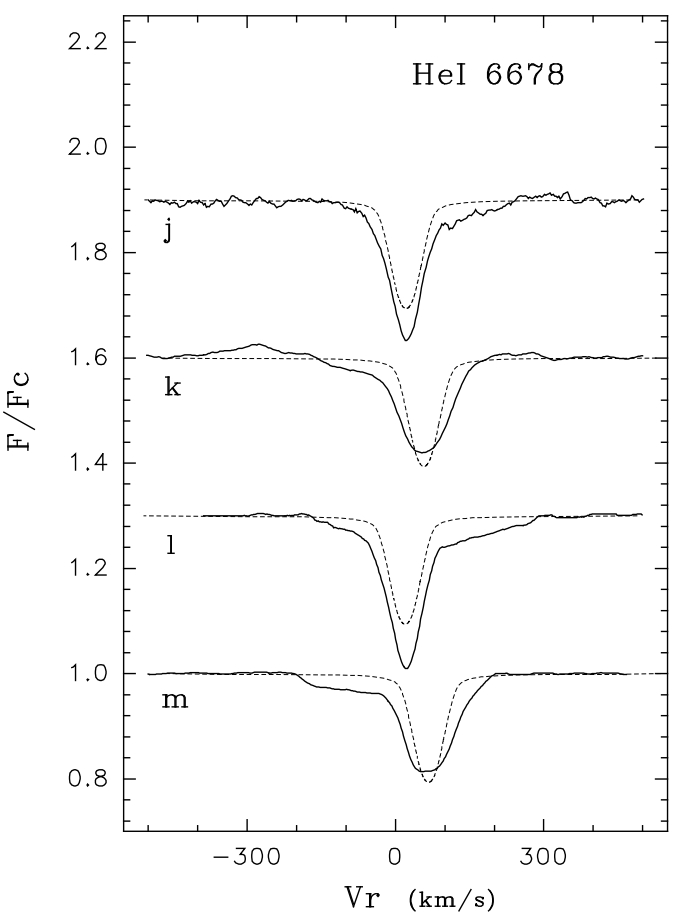

Fig. 5. Typical profiles of the He I $\lambda 6678 \AA$ line observed on following dates: j) Nov. 1, 2003; k) Dec.22, 2003; l) Oct. 25, 2004; m) Jan. 13, 2005. Two absorption components (a deep and a shallow) changing their velocity positions in counter-phase are clearly seen. The synthetic photospheric profile centered at the axis of symmetry of the narrow deep component is given for comparison. The velocity scale is given in the heliocentric rest frame.

was found to be close to half a year forced us to be cautious and test this estimation more carefully. A search for the period in separate data, obtained from March 1999 to March 2003 (36 points) and from October 2003 to March 2005 (40 points) with the Lafler \& Kinman (1965) method implemented in the computer code by Pelt (1980) resulted in values close to the one obtained for both observing seasons $\left(P=181^{\mathrm{d}} .8 \pm 1.2\right.$ and $P=185^{\mathrm{d}} .4 \pm 3^{\mathrm{d}} .3$ respectively).

Figure 7 illustrates the common phase curve shown in Fig. 6 superimposed with the observational data for these two seasons (the upper and middle panels respectively). One can see that the phase coverage in the first case is incomplete. Only the part of the curve placed below the $\gamma$-velocity can be reconstructed satisfactorily. The 2003-2005 data are distributed more evently over phases, and the phase coverage in this case is practically complete. The bottom panel of Fig. 7 illustrates the data obtained at LNA on May 11, 2000 and the 1994-1998 data obtained at OHP and published by Corporon \& Lagrange (1999) folded with the 183.34-period and superposed with our phase curve. Almost all velocities except two OHP points correspond to the curve. These two measurements were carried out on Julian dates JD 2450621.67 and JD 2450622.73 ; the given values of the radial velocities are sufficiently lower than those that would be predicted from our orbital curve: 36.0 and $35.1 \mathrm{~km} \mathrm{~s}^{-1}$, respectively, compared to the expected $50-60 \mathrm{~km} \mathrm{~s}^{-1}$. The profiles of the $\mathrm{He} \mathrm{I} \lambda 6678 \AA$ line on these dates, presented by Corporon $\&$ Lagrange (1999, top of Fig. 6), are very asymmetric and much shallower than the other profiles of the same line given in that figure. Their relative intensity at the minimum is about 0.90 in comparison with $0.75-0.85$ observed in our spectra and 0.80 for the synthetic profile (Fig. 5). The photospheric component of

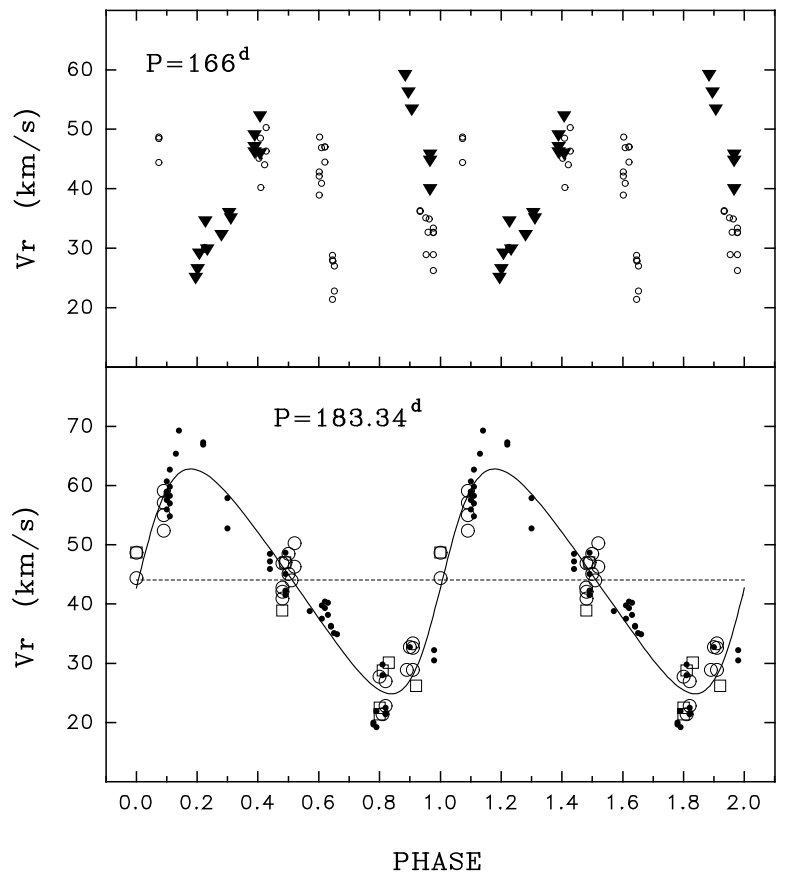

Fig. 6. Top: obtained radial velocities (open circles) of HD 53367 from data collected at the CrAO (from March 1999 to March 2003), folded with the period $P=166$ days suggested by Corporon \& Lagrange (1999) superposed to the data used by these authors (filled triangles) to obtain this period. The new data do not match the period estimated by them. Bottom: the radial velocities of the star measured at the CrAO during the whole observing period (1999-2005) folded with the period $P=183.34$ days. The least-squares fit to the observations indicated by the solid line illustrates the calculated orbital curve. The initial phase corresponds to the epoch of periastron passage. The dashed line indicates the $\gamma$-velocity. The following symbols are used for different spectral lines: filled circles - He I 5876, 6678 A lines, open circles O II $6641 \AA$, and open squares $-\mathrm{H} \alpha$ and $\mathrm{H} \beta$ (when the envelope was practically dissipated).

Table 3. Orbital solution obtained for HD 53367. All values are given with errors $( \pm \sigma)$.

\begin{tabular}{ccc}
\hline \hline Orbital elements & CrAO data & CrAO + LNA + OHP data \\
\hline$P$ (days) & $183.34 \pm 0.45$ & $183.70 \pm 0.10$ \\
$T_{0}$ (days) & $2449127.90 \pm 9.76$ & $2449118.71 \pm 2.86$ \\
$a_{1} \sin i\left(R_{\odot}\right)$ & $62 \pm 3$ & $60 \pm 2$ \\
$e$ & $0.25 \pm 0.04$ & $0.28 \pm 0.03$ \\
$\omega($ degr $)$ & $266.6 \pm 8.0$ & $263.8 \pm 6.6$ \\
$K_{1}\left(\mathrm{~km} \mathrm{~s}^{-1}\right)$ & $19.0 \pm 0.7$ & $18.6 \pm 0.7$ \\
$\gamma\left(\mathrm{km} \mathrm{s}^{-1}\right)$ & $44.1 \pm 0.5$ & $44.2 \pm 0.5$ \\
$f\left(M_{2}\right)\left(M_{\odot}\right)$ & $0.100 \pm 0.016$ & $0.107 \pm 0.013$ \\
\hline
\end{tabular}

where: $P$ - orbital period; $T_{0}-$ the periastron epoch; $a_{1}-$ the semimajor axis of the primary component; $i-$ the inclination angle of the orbit to the line-of-sight; $e$ - the orbital eccentricity; $\omega$ - the periastron longitude; $K_{1}$ - the semi-amplitude of the orbital curve of the primary component; $\gamma$ - the radial velocity of the system mass centre; $f\left(M_{2}\right)$ the mass function of the secondary component.

these profiles is strongly overlapped by CS emission that makes it unsuitable for radial velocity measurements.

The existence of a systematic $\sim 0.04$ phase shift can be suspected for the OHP data obtained 5-7 years earlier than our observations. This effect is probably connected with the fact that the accuracy of the 183.3-period estimation based only on 


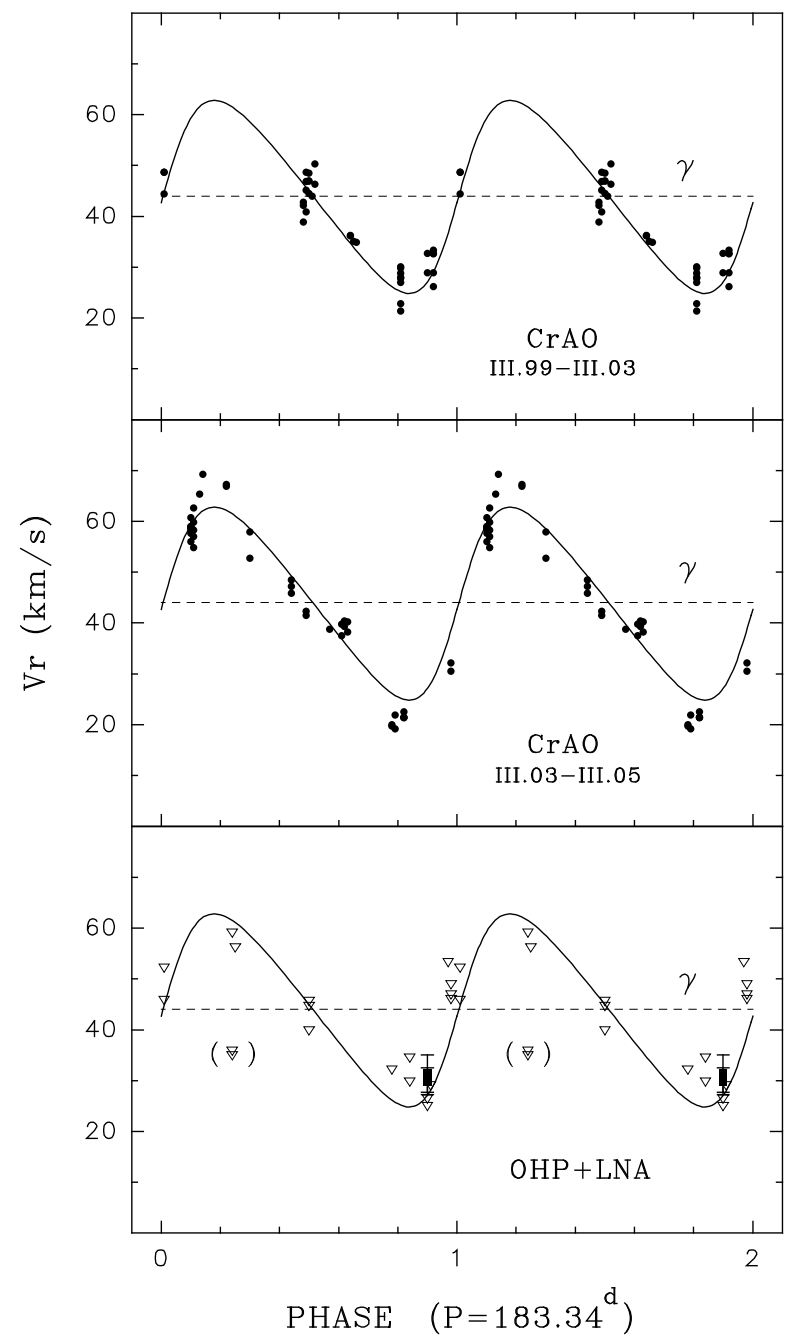

Fig. 7. Examination of correctness of the calculated orbital curve displayed in Fig. 6 on the basis of data obtained at different observatories and seasons. Top: the common orbital curve marked by solid line with superimposed radial velocities obtained at CrAO only from March 1999 to March 2003. Middle: the same as in the upper panel for data obtained from October 2003 to March 2005. Bottom: the same as in the upper panel for the OHP data by Corporon \& Lagrange (1999) obtained in 1994-1998 (open triangles) and from the LNA-data obtained on May 11, 2000 (filled squares). Two LNA-points are presented with the corresponding error bars (see text). Two OHP points which are not in agreement with the orbital curve are given in brackets.

our 1999-2005 data is not enough to extrapolate the calculated orbital curve to a time about 1500 days earlier.

Because of that, we calculated a refined orbital solution based on all data obtained at the CrAO, LNA and OHP, excluding the two OHP points corresponding to the dates JD 2450621.67 and JD 2450622.73 (95 points). The refined values of the orbital elements are given in the third column of Table 3. Figure 8 illustrates the temporal spread of all available data obtained at OHP, CrAO and LNA from 1994 to 2005 in comparison with the obtained orbital curve.

\section{Discussion}

\subsection{Nature of the secondary component}

Inspecting the variable He I $\lambda 6678 \AA$ line profiles presented in Fig. 5 we see a wide shallow absorption component changing
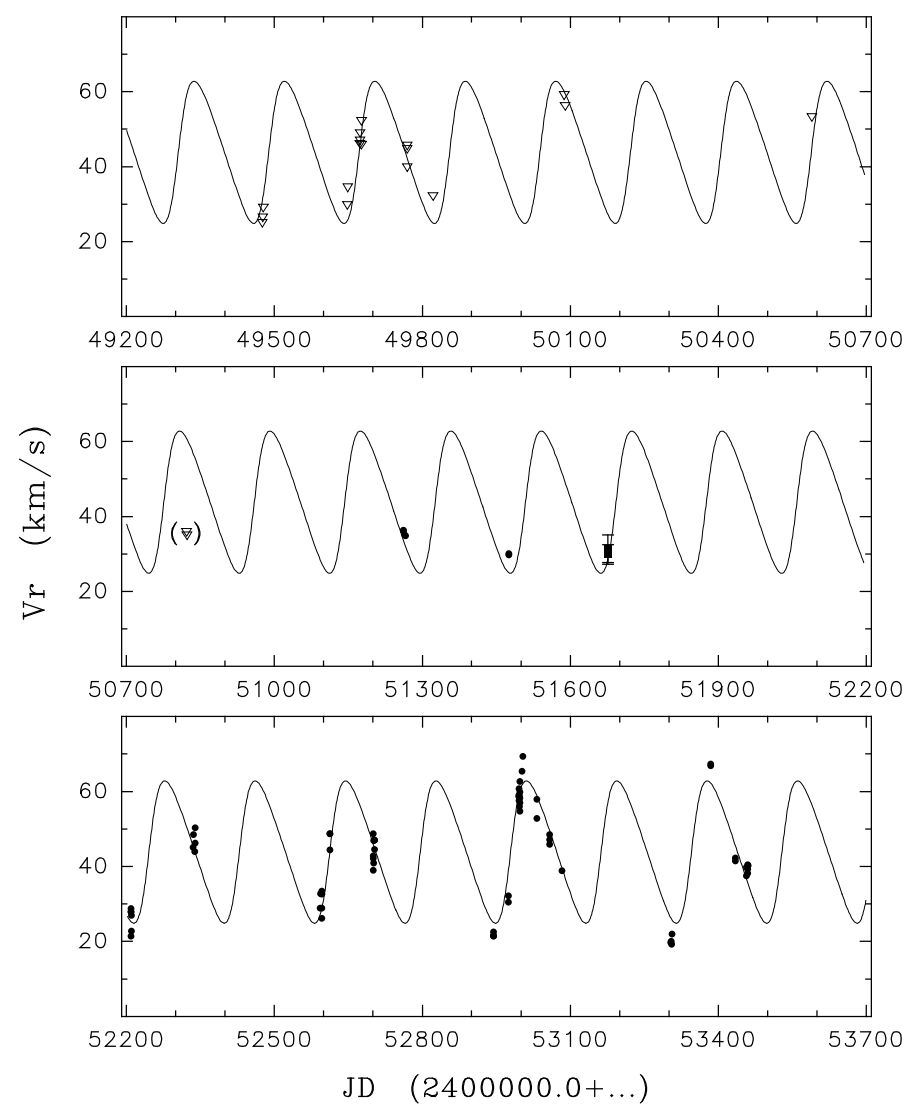

Fig. 8. The temporal spread of radial velocities of the star observed from 1994 to March 2005 in comparison with the orbital curve calculated for $P=183.70$ days. The symbols are the same as in Fig. 7 .

its position in counterphase with the main deep absorption. This feature may be the line in the spectrum of the secondary system's component rotating more rapidly then the primary one ( $v \sin i \approx 150 \mathrm{~km} \mathrm{~s}^{-1}$ ). However, the equivalent width of the feature and the amplitude of its positional variability are comparable with those of the main component. Hence, the masses of both components must be comparable too. We calculated a series of synthetic spectra of the system containing a primary star with fixed parameters $\left(T_{\mathrm{eff}}=30000 \mathrm{~K}, \log g=4.2\right.$, and $v \sin i=$ $40 \mathrm{~km} \mathrm{~s}^{-1}$ ) and a secondary star with parameters varying in the following ranges: $T_{\text {eff }}=30000-20000 \mathrm{~K}, \log g=3.5-4.5$, $v \sin i=160 \mathrm{~km} \mathrm{~s}^{-1}$. The spectra were constructed in the spectral region 4575-4725 $\mathrm{A}$, where our LNA spectrum shows many photospheric lines with a minimum of CS influence (Fig. 3). The comparison of the synthetic combined spectra with the observed one shows that an accordance between them is impossible. If the components are different in spectral class, additional lines of the lower temperature star would appear in the combined spectrum, and if the $T_{\text {eff }}$ of the secondary is close to that of the primary, the deep narrow absorptions in the calculated spectrum would be sufficiently lower in depth to be seen in the observed spectrum, because of a significant contribution of the secondary to the emission in the continuum. Therefore, we concluded that the mass of the system's secondary is likely to be much smaller than that of the primary.

Thus we concluded that the origin of the wide shallow absorption component of the He I $\lambda 6678 \AA$ line was likely to be related to the circumstellar matter.

The mass of the secondary component can be estimated from its mass function $f\left(M_{2}\right)$, given in Table 3 , if the angle of its 


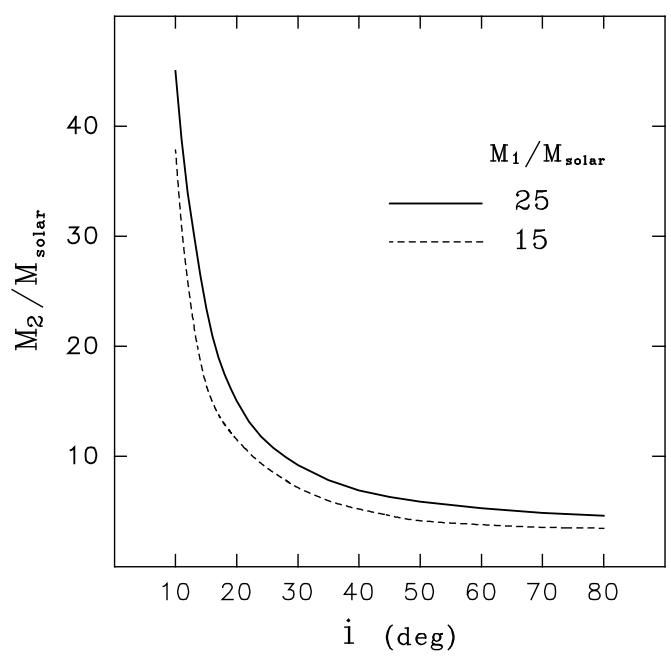

Fig. 9. Dependence of the mass of the secondary star, $M_{2}$, on the orbital angle, $i$, calculated for two boundary estimations of the mass of the primary star, $M_{1}$, using the mass function, $f\left(M_{2}\right)$, indicated in Table 3.

orbital inclination $i$ is known. Figure 9 gives the relationship between $i$ and $M_{2}$ calculated for two values of the primary component's mass $M_{1}$. The curves show that the higher the inclination angle, the lower the mass of the secondary component. In the range of $i \geq 50^{\circ}$ the $M_{2}$ becomes gradually insensitive to the $i$ variation and corresponds to about $4-5 M_{\odot}$.

We suppose that: a) most of the CS matter around HD 53367 is collected in an equatorial disk, and b) this disk is likely to be coplanar with the orbital plane of the system. The former statement is based on the fact that the contribution of the stellar wind in the formation of the CS line profiles in the object's spectrum is much smaller than that of the disk. This is confirmed by the absence of obvious signs of matter outflow in the UV resonance $\mathrm{Mg}$ II $h$ and $k$ doublet which is a solid indicator of stellar wind (Imhoff 1994). The second assertion follows from the current evolutionary scenario for binary systems.

The inclination angle of the orbit of HD 53367 cannot be very close to $90^{\circ}$ because no specific photometric variability, typical for objects with an edge-on oriented disk and connected with the motion of dusty clouds near the equatorial plane, has been observed in this star. Nevertheless, there is indirect evidence that this angle is not small either. Some evidence comes from the previous investigations of HD 53367. Oegerle et al. (1983) gave indications against a "pole-on" orientation of the object. They argued that the simultaneous occurrence of emission in the O I $\lambda 8446 \AA$ line and of absorption in the O I $\lambda 1302 \AA$ line can only be understood if the object has a disk-like envelope, which is observed at a large inclination angle with the polar axis. Later, Oudmaijer \& Drew (1999) carried out spectropolarimetric observations of HD 53367 over the $\mathrm{H} \alpha$ profile and concluded that the circumstellar envelope projected on the sky is seen as a flattened feature.

Also, an evidence in favour of a large $i$ is given by the results of our spectroscopy. The spatial distribution of circumstellar gas collected close to the orbital plane and emitting in the $\mathrm{H} \alpha$ and $\mathrm{H} \beta$ lines is likely to be complex, but, according to general physics, the velocities of its large-scale motion near the stellar surface of the primary component have to be comparable with those for a rotating Keplerian disk. For the mass $M=20 M_{\odot}$ and the radius $R=7.5 R_{\odot}$ the value of this velocity is about $700 \mathrm{~km} \mathrm{~s}^{-1}$. The width of the observed emission wings of $\mathrm{H} \alpha$ and $\mathrm{H} \beta$ profiles in time when these lines are strong or are forming corresponds to this value (see Fig. 2, dates "a", "b", and " $\mathrm{j}$ "). Therefore the orbital inclination angle $i$ has to be large enough for $\sin i$ to be close to 1 .

Taking into account all the reasons given above, we conclude that the angle $i \geq 50^{\circ}$ and the mass of the second component of the system $M_{2}$ is likely to be $4-5 M_{\odot}$. A young object of such mass has to be a pre-main sequence Herbig Be star with $T_{\text {eff }}=$ 10 000-15 000 K (Palla \& Stahler 1993).

\subsection{Circumstellar activity in the system}

We suggest that changes of the amount of the emitting gas in the circumstellar envelope around HD 53367 are responsible for the observed photometric variability of this object. Taking into account the interstellar reddening $\left(A_{V}=2 \mathrm{~m} 3, R=3.14\right.$, Tjin A Djie et al. 2001), HD 53367 has, in the low-brightness state, colors usual for a typical B0 v star: $B-V=-0.35$; $V-R=-0$. 17 . As the object increases in brightness its colors become redder. The mean differences of magnitude between the bright and the low-brightness photometric states are $\Delta B=0 \mathrm{~m} 14$, $\Delta V=0.26, \Delta R=0.43$. The spectrum of the superimposed emission corresponds to the spectrum of the emitting hydrogen gas of intermediate optical thickness. We carried out a rough estimation of the parameters of this gas. Its temperature was taken to be constant $\left(T_{\mathrm{e}}=20000 \mathrm{~K}\right)$ throughout the envelope, since circumstellar He I line components are clearly seen in the object's spectrum. As a simplified model of the envelope we considered a Keplerian disk oriented edge-on with a geometric thickness $2 R_{*}$. As a result we obtained that the effective optical thickness of the gas at wavelengths corresponding to the $B, V, R$-bands was respectively $0.13,0.21$ and 0.38 . The estimation of the electron density near the surface of the primary, obtained as the mean value from calculations for different wavelengths, was $N_{\mathrm{e}}=1.65 \pm 0.12 \times 10^{12} \mathrm{~cm}^{-3}$.

Variations of the amount of the emitting circumstellar gas near HD 53367 take place in the form of episodic dissipation of its envelope, followed by the appearance of a new envelope. Both these processes start near the surface of the primary and then spread to the outlying part of the circumstellar space. At the stage of dissipation the internal envelope boundary shifts outward from the star, and the disk transforms into a circumstellar ring. At this time another new envelope forms near the stellar surface. This scenario explains the behaviour of the emission in $\mathrm{H} \alpha$ and $\mathrm{H} \beta$ at a time when the brightness decreases. As it was shown in Sect. 3.1 the fall of the emission in lines occurs more slowly than in the continuum. This is because the sensitivity of the gas emission in the continuum to electron density changes is much greater than the emission in lines. Model calculations show that the square of the electron density, which is a determining factor for the emission intensity in the continuum, drops with the distance from the star more quickly than the abundances of excited atomic levels responsible for the emission in lines (Pogodin 1986). As a result, a circumstellar gaseous ring empty on the inside can emit substantially in lines and be undetectable in the continuum.

According to this scenario, the appearance of a new envelope has to be followed by simultaneous strengthening of the brightness as well as the emission in lines proceeding on similar timescales. Forthcoming spectroscopic and photometric investigations of HD 53367 covering this period of time may confirm this scenario.

The results of our spectroscopy allow us to draw some inferences about the spatial structure of the circumstellar matter in the binary system HD 53367. In Sect. 3.2 we discussed 


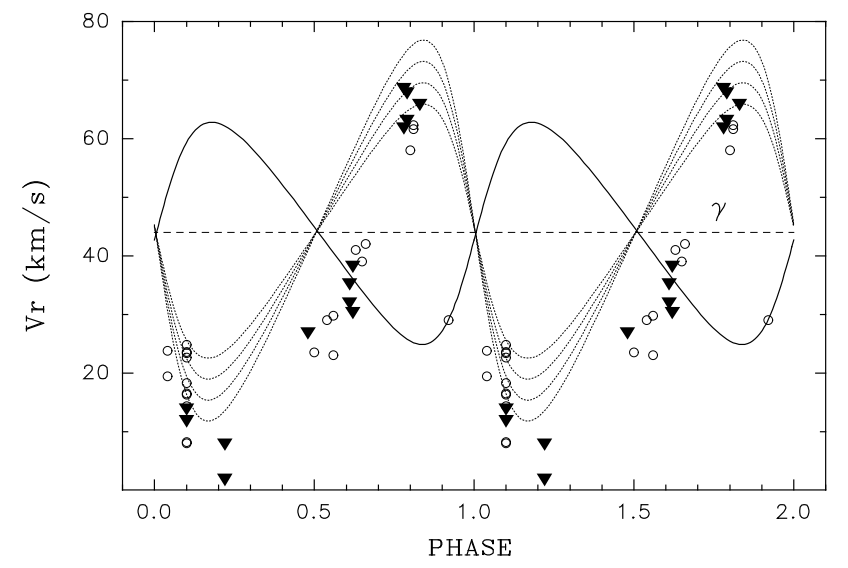

Fig. 10. Radial velocities of different features originating in the circumstellar media in comparison with the orbital curve for the primary star (solid line). A set of expected curves of the secondary component calculated for different masses and illustrating the typical shape of the curves is marked by dotted lines. Filled triangles represent the velocities obtained from the shallow absorption component of He I $\lambda 6678 \AA$, the open circles give the center of gravity of the emission $\mathrm{H} \alpha$ and $\mathrm{H} \beta$ profiles. The $\gamma$-velocity is indicated by the dashed line.

the behaviour of the wide shallow absorption component of the He I $\lambda 6678 \AA$ line, changing its velocity position in counterphase with the velocity of the primary component (Fig. 5). A conclusion has been drawn regarding the circumstellar origin of this feature. Figure 10 illustrates its variations with the orbital phase in comparison with velocities corresponding to the gravity centre of the emission $\mathrm{H} \alpha$ and $\mathrm{H} \beta$ profiles placed above their photospheric background. These velocities were measured as the bisector velocities of the wide component at the 0.98 intensity level. These velocities are arranged in a common phase curve which clearly demonstrates variations in counter-phase with the orbital curve of the primary star. A set of orbital curves that can be expected for the low-mass secondary component is plotted in Fig. 10. The phase curve constructed for the circumstellar features is sufficiently different in shape compared to the curves from this set. Its behaviour means that the circumstellar matter is not rigidly bound to either component of the system, but forms a common extended envelope and exhibits a spacial asymmetry with a larger concentration toward the secondary component. Such a structural property of the envelope around HD 53367 is not surprising taking into account the dissipative influence of hot massive stars (like the primary star of our system) onto the circumstellar matter in their vicinity.

The structure of the multicomponent profile of the He I $\lambda 6678 \AA$ line in the spectrum of HD 53367 is very similar to that of the $\mathrm{He} \mathrm{I} \lambda 5875 \AA$ line profile in the spectrum of another eccentric binary system, HD 200775, including a deep narrow absorption, a shallow wide absorption and a blueshifted emission peak (Pogodin et al. 2000, Fig. 2). Similar to HD 53367, that system consists of a massive primary (B2e) and a lowermass $\left(M \approx 3-4 M_{\odot}\right)$ secondary component. Its eccentricity $e$ is also about 0.3 (Pogodin et al. 2004), and the orbital inclination angle is large (about $70^{\circ}$ ).

The concrete physical mechanisms initiating the circumstellar activity observed in HD 53367 remain to be determined. The source of dissipation of the circumstellar matter around hot B0 stars is well-known. It is the stellar radiation and the matter outflow from the star. But the nature of the appearance of a new dense gaseous envelope near the star is more puzzling. Two different possibilities can be considered: a) a sudden infall of the circumstellar matter from the remote part of the envelope into its internal region; and b) activity of the star itself.

Some signatures of the motion of the CS matter toward the star are observed on dates when the envelope is not yet dissipated ("a", "b", and "c"). The V/R ratio of $\mathrm{H} \alpha$ and $\mathrm{H} \beta$ profiles was more than 1 , and the velocity of the absorption component demonstrated a small positive shift relative to the system velocity derived from photospheric lines $\left(+5-6 \mathrm{~km} \mathrm{~s}^{-1}\right)$. Nevertheless, no obvious large-scale matter infall event has been observed for the object during our study.

If the variations taking place in the circumstellar envelope are of stellar origin, the activity observed in HD 53367 resembles the well-known Be phenomenon with the specific alternation of $\mathrm{B}-\mathrm{Be}$ states of the object. We can assume that, in spite of the youth of HD 53367, the massive and quickly evolving primary star has already passed through the main sequence stage and is now a classical Be star. At the same time the secondary component still remains a pre-main sequence object. In Fig. 1 a small local jump of brightness was observed at the end of 1999 (JD $2451488-J D 2451$ 529). Just before the jump, on October 24, 1999 (JD 2 451 476) the double-peaked $\mathrm{H} \alpha$ profile showed a $V / R$-ratio smaller than 1 . On all other dates of our observations this ratio was always $\geq 1$. The fact that $V / R<1$ occurred immediately before the brightness jump is an indicator of matter outflow from the star resulting in a short-term strengthening of the gaseous envelope. This supports the hypothesis of the stellar activity as a trigger of changes in the circumstellar envelope.

\section{Conclusions}

High-resolution spectroscopic observations of the young massive B0e star HD 53367 carried out from 1999-2005 contribute to a better understanding of some remarkable properties of this object. Our observations covered a period when the object passed to its low-brightness state and stayed in this state.

We have confirmed that the photometric activity of HD 53367 is the result of episodic dissipation followed by new appearances of its gaseous circumstellar envelope. At the time of maximum development of the envelope, the initial electron density of the emitting gas was estimated to be $N_{\mathrm{e}} \sim 10^{12} \mathrm{~cm}^{-3}$.

We have re-estimated the period of the cyclic radial velocity variations observed for several photospheric lines in the object's spectrum. A new value of $P \approx 183^{\mathrm{d}}$ was obtained.

Assuming the binary nature of HD 53367 we have determined its orbital elements and stellar parameters. The system consists of a massive $\left(\sim 20 M_{\odot}\right)$ primary B0e star in the main sequence (if not later) stage and a pre-main sequence secondary object of $M \approx 4-5 M_{\odot}$ with a main distance $\sim 1.7$ AU between them. The orbital eccentricity was estimated to be $e=0.28$.

The circumstellar matter around the system forms a common envelope with a dominant concentration toward the secondary component.

We have discussed the possible origin of the active phenomena observed in HD 53367. One of the possibilities is that, in spite of the system being young, the quickly evolved primary B0e star has already become a classical Be star exhibiting a specific alternation of the B-Be stages.

Future spectroscopic and photometric observations near a new passage of this object to its bright state will be most useful to understand the properties of this system and its components. 
Acknowledgements. We are grateful to P. Corporon for providing access to his spectroscopic database and to K. Grankin for acquainting us with recent photometric data obtained at the Mt. Maidanak Observatory. G.A.P.F. acknowledges a grant from the Brazilian research agency $\mathrm{CNPq}$.

\section{References}

Claria, J. J. 1974, AJ, 79, 1022

Corporon, P., \& Lagrange, A.-M. 1999, A\&AS, 136, 429

Finkenzeller, U., \& Mundt, R. 1984, A\&AS, 55, 109

Herbig, G. H. 1960, ApJS, 4, 337

Imhoff, C. L. 1994, in The Nature and Evolutionary Status of Herbig Ae/Be Stars, ASP Conf. Ser., 62, 107
Lafler, J., \& Kinman, T. D. 1965, ApJS, 11, 216

Oegerle, W. R., Peters, G. J., \& Polidan, R. S. 1983, PASP, 95, 147

Oudmaijer, R. D., \& Drew, J. E. 1999, MNRAS, 305, 166

Palla, F., \& Stahler, S. W. 1993, ApJ, 418, 414

Pelt, J. 1980, in Frequency analysis of astronomical time sequences, Valgus, Tallin

Piskunov, N. E. 1992, in Stellar Magnetism, 92

Pogodin, M. A. 1986, Astrofizika, 24, 279

Pogodin, M. A., Miroshnichenko, A. S., Bjorkman, K. S., Morrison, N. D., \& Mulliss, C. L. 2000, A\&A, 359, 299

Pogodin, M. A., Miroshnichenko, A. S., Tarasov, A. E., et al. 2004, A\&A, 417, 715

Tjin A Djie, H. R. E., van den Ancker, M. E., Blondel, P. F. C., et al. 2001, MNRAS, 325, 1441

Tokovinin, A. 1992, in Complementary Approaches to Double and Multiple Star Research, IAU Colloq., 135, ASP Conf. Ser., 32,573 
M. A. Pogodin et al.: The Herbig B0e star HD 53367, Online Material p 1

\section{Online Material}


M. A. Pogodin et al.: The Herbig B0e star HD 53367, Online Material p 2

Table 1. Observation log for spectroscopic data collected at CrAO (Ukraine). A total of 105 spectra of HD 53367 were gathered during 36 obseving nights from March 1999 to March 2005.

\begin{tabular}{|c|c|c|c|c|c|c|c|c|c|}
\hline$\overline{\text { Date }}$ & $\begin{array}{c}\text { JD } \\
2400000+\end{array}$ & Lines & $\begin{array}{c}S / N \text { at } \\
\text { the } F_{\mathrm{c}} \text { level }\end{array}$ & $\begin{array}{c}V_{\mathrm{r}} \\
\mathrm{km} \mathrm{s}^{-1}\end{array}$ & $\overline{\text { Date }}$ & $\begin{array}{c}\text { JD } \\
2400000+\end{array}$ & Lines & $\begin{array}{c}S / N \text { at } \\
\text { the } F_{\mathrm{c}} \text { level }\end{array}$ & $\begin{array}{c}V_{\mathrm{r}} \\
\mathrm{km} \mathrm{s}^{-1}\end{array}$ \\
\hline \multirow[t]{4}{*}{ Mar. 23, 99} & 51261.23 & $\mathrm{H} \alpha$ & 130 & & Nov. 30,03 & 52974.44 & $\mathrm{He}$ I $\lambda 6678$ & 85 & 32.2 \\
\hline & 51261.24 & $\mathrm{He}$ I $\lambda 5876+\mathrm{Na}$ I & 130 & 36.3 & & 52944.45 & Не I $\lambda 6678$ & 85 & 30.5 \\
\hline & 51261.26 & $\mathrm{H} \beta$ & 35 & & Dec. 22,03 & 52996.44 & $\mathrm{H} \alpha$ & 75 & \\
\hline & 51261.28 & Не г $\lambda 6678$ & 125 & 36.2 & & 52996.45 & $\mathrm{H} \alpha$ & 75 & \\
\hline \multirow[t]{3}{*}{ Mar. 26, 99} & 51264.21 & $\mathrm{H} \alpha$ & 110 & & & 52996.47 & Не I $\lambda 6678$ & 75 & 59.0 \\
\hline & 51264.23 & $\mathrm{He}$ I $\lambda 5876+\mathrm{Na}$ I & 100 & & & 52996.48 & He I $\lambda 6678$ & 70 & 58.6 \\
\hline & 51264.24 & Не I $\lambda 6678$ & 120 & 35.1 & & 52996.50 & O II $\lambda 6641$ & 70 & 59.1 \\
\hline \multirow[t]{3}{*}{ Mar. 28, 99} & 51266.23 & $\mathrm{H} \alpha$ & 90 & & & 52996.52 & O II $\lambda 6641$ & 70 & 57.1 \\
\hline & 51266.25 & $\mathrm{He}$ I $\lambda 5876+\mathrm{Na}$ I & 85 & 38.3 & & 52996.53 & He I $\lambda 6678$ & 65 & 60.7 \\
\hline & 51266.28 & Не I $\lambda 6678$ & 90 & 34.9 & & 52996.55 & Не I $\lambda 6678$ & 65 & 57.6 \\
\hline \multirow[t]{3}{*}{ Oct. 24,99} & 51475.56 & $\mathrm{H} \alpha$ & 65 & & & 52996.56 & Не I $\lambda 6678$ & 65 & 58.9 \\
\hline & 51475.58 & $\mathrm{H} \beta$ & 35 & 30.1 & & 52996.58 & He I $\lambda 6678$ & 65 & 58.6 \\
\hline & 51475.60 & $\mathrm{He} \mathrm{I} \lambda 5876+\mathrm{Na} \mathrm{I}$ & 30 & 29.8 & & 52996.59 & Не I $\lambda 6678$ & 60 & 56.0 \\
\hline \multirow[t]{5}{*}{ Oct. 26,01} & 52209.47 & $\mathrm{H} \alpha$ & 65 & & & 52996.61 & $\mathrm{H} \alpha$ & 55 & \\
\hline & 52209.49 & $\mathrm{He}$ I $\lambda 5876+\mathrm{Na}$ I & 70 & 28.0 & & 52996.62 & $\mathrm{H} \alpha$ & 30 & \\
\hline & 52209.50 & $\mathrm{H} \beta$ & 30 & 28.8 & Dec. 23,03 & 52997.38 & $\mathrm{H} \alpha$ & 80 & \\
\hline & 52209.56 & O II $\lambda 6641$ & 90 & 21.4 & & 52997.39 & $\mathrm{H} \alpha$ & 75 & \\
\hline & 52209.58 & О II $\lambda 6641$ & 90 & 27.8 & & 52997.41 & $\mathrm{H} \alpha$ & 75 & \\
\hline \multirow[t]{3}{*}{ Oct. 27, 01} & 52210.48 & $\mathrm{H} \alpha$ & 80 & & & 52997.42 & O II $\lambda 6641$ & 70 & 52.4 \\
\hline & 52210.50 & O II $\lambda 6641$ & 90 & 22.8 & & 52997.44 & O II $\lambda 6641$ & 75 & 55.0 \\
\hline & 52210.53 & O II $\lambda 6641$ & 90 & 27.0 & & 52997.46 & He I $\lambda 6678$ & 80 & 58.3 \\
\hline Mar. 01, 02 & 52335.22 & O II $\lambda 6641$ & 100 & 45.1 & & 52997.47 & $\mathrm{He}$ I $\lambda 6678$ & 75 & 57.0 \\
\hline \multirow[t]{2}{*}{ Mar. 02, 02} & 52336.21 & O II $\lambda 6641$ & 70 & 48.5 & & 52997.49 & Не I $\lambda 6678$ & 70 & 54.8 \\
\hline & 52336.31 & $\mathrm{H} \alpha$ & 40 & & & 52997.50 & $\mathrm{H} \alpha$ & 65 & \\
\hline Mar. 04, 02 & 52338.33 & $\mathrm{He}$ I $\lambda 5876+\mathrm{Na}$ I & 35 & 44.0 & & 52997.51 & $\mathrm{H} \alpha$ & 75 & \\
\hline \multirow[t]{2}{*}{ Mar. 05, 02} & 52339.20 & O II $\lambda 6641$ & 90 & 46.3 & & 52997.53 & $\mathrm{H} \alpha$ & 70 & \\
\hline & 52339.23 & O II $\lambda 6641$ & 80 & 50.3 & & 52997.55 & $\mathrm{H} \alpha$ & 65 & \\
\hline Nov. 14, 02 & 52592.60 & O II $\lambda 6641$ & 80 & 28.9 & & 52997.57 & $\operatorname{He} \mathrm{I} \lambda 6678$ & 60 & 62.7 \\
\hline Nov. 15,02 & 52593.56 & O II $\lambda 6641$ & 100 & 32.7 & & 52997.58 & He I $\lambda 6678$ & 55 & 59.8 \\
\hline \multirow[t]{6}{*}{ Nov. 18,02} & 52596.46 & $\mathrm{H} \alpha$ & 50 & & Dec. 28,03 & 53002.50 & Не I $\lambda 6678$ & 55 & 65.4 \\
\hline & 52596.49 & $\mathrm{He}$ I $\lambda 5876+\mathrm{Na}$ I & 60 & 32.7 & Dec. 29,03 & 53003.52 & Не I $\lambda 6678$ & 50 & 69.3 \\
\hline & 52596.53 & O II $\lambda 6641$ & 85 & 32.6 & Jan. 27, 04 & 53032.41 & He I $\lambda 6678$ & 30 & 52.8 \\
\hline & 52596.56 & O II $\lambda 6641$ & 90 & 28.9 & & 53032.43 & $\operatorname{He}$ I $\lambda 6678$ & 50 & 57.9 \\
\hline & 52596.58 & O II $\lambda 6641$ & 90 & 33.4 & Feb. 22, 04 & 53058.29 & He I $\lambda 6678$ & 35 & 47.2 \\
\hline & 52596.62 & $\mathrm{H} \beta$ & 30 & 26.2 & & 53058.31 & Не I $\lambda 6678$ & 55 & 47.9 \\
\hline \multirow[t]{3}{*}{ Dec. 04, 02} & 52612.53 & $\mathrm{H} \alpha$ & 60 & 48.7 & & 53058.34 & He I $\lambda 6678$ & 55 & 48.5 \\
\hline & 52612.56 & O II $\lambda 6641$ & 85 & 44.4 & Mar. 13, 04 & 53078.22 & $\mathrm{H} \alpha$ & 45 & \\
\hline & 52612.59 & O II $\lambda 6641$ & 85 & 48.7 & Mar. 18, 04 & 53083.37 & Не I $\lambda 6678$ & 35 & 38.8 \\
\hline \multirow[t]{4}{*}{ Mar. 01, 03} & 52700.21 & $\mathrm{H} \alpha$ & 60 & 38.9 & Oct. 25,04 & 53303.53 & $\mathrm{He}$ I $\lambda 6678$ & 80 & 19.7 \\
\hline & 52700.23 & O II $\lambda 6641$ & 70 & 42.8 & Oct. 26,04 & 53304.54 & He I $\lambda 6678$ & 110 & 20.0 \\
\hline & 52700.26 & O II $\lambda 6641$ & 85 & 42.1 & Oct. 27,04 & 53305.54 & $\mathrm{He}$ I $\lambda 6678$ & 75 & 19.2 \\
\hline & 52700.29 & $\mathrm{He}$ I $\lambda 5876+\mathrm{Na}$ I & 70 & 48.7 & Oct. 28,04 & 53306.53 & Hе I $\lambda 6678$ & 90 & 21.9 \\
\hline \multirow[t]{2}{*}{ Mar. 02, 03} & 52701.32 & О II $\lambda 6641$ & 90 & 40.9 & Jan. 13, 05 & 53384.38 & Не I $\lambda 6678$ & 35 & 67.3 \\
\hline & 52701.35 & O II $\lambda 6641$ & 100 & 46.9 & & 53384.42 & Не I $\lambda 6678$ & 55 & 66.9 \\
\hline \multirow[t]{3}{*}{ Mar. 04, 03} & 52703.35 & O II $\lambda 6641$ & 70 & 47.0 & Mar. 04, 05 & 53434.34 & Не г $\lambda 6678$ & 65 & 42.3 \\
\hline & 52703.37 & $\mathrm{He}$ I $\lambda 5876+\mathrm{Na}$ I & 40 & 44.5 & & 53434.39 & Не I $\lambda 6678$ & 50 & 41.5 \\
\hline & 52703.39 & $\mathrm{H} \alpha$ & 50 & 47.0 & Mar. 27, 05 & 53457.25 & Не I $\lambda 6678$ & 70 & 37.6 \\
\hline \multirow{6}{*}{ Nov. 01,03} & 52944.54 & $\mathrm{H} \alpha$ & 65 & & & 53457.29 & He I $\lambda 6678$ & 55 & 39.8 \\
\hline & 52944.55 & Не I $\lambda 6678$ & 75 & 21.5 & Mar. 29, 05 & 53459.24 & Не I $\lambda 6678$ & 60 & 39.3 \\
\hline & 52944.57 & He I $\lambda 6678$ & 85 & 21.6 & & 53459.29 & $\mathrm{He}$ I $\lambda 6678$ & 65 & 40.4 \\
\hline & 52944.59 & He I $\lambda 5876+\mathrm{Na}$ I & 65 & & & 53459.33 & Не I $\lambda 6678$ & 50 & 38.2 \\
\hline & 52944.61 & $\mathrm{H} \beta$ & 30 & 21.4 & Mar. 30, 05 & 53460.26 & Не I $\lambda 6678$ & 45 & 40.2 \\
\hline & 52944.64 & $\mathrm{H} \beta$ & 35 & 22.5 & & & & & \\
\hline
\end{tabular}

The heliocentric Julian date (JD) is given for the middle point of the exposure; the signal-to-noise ratio $(S / N)$ corresponds to the continuum level $F_{\mathrm{c}}$. In the last column the radial velocity of the star is given for lines which were chosen for these estimations. 